\title{
Antioxidant, radical-scavenging, anti-inflammatory, cytotoxic and antibacterial activities of methanolic extracts of some Hedyotis species
}

\begin{abstract}
The antioxidant, radical-scavenging, anti-inflammatory, cytotoxic and antibacterial activities of methanolic extracts of seven Hedyotisspecies were investigated. The antioxidant activity was evaluated by the ferric thiocyanate (FTC) and thiobarbituric acid (TBA) methods while the radical scavenging activity was measured by the 1,1-diphenyl-2-picrylhydrazyl (DPPH) method. The anti-inflammatory activity related to NO inhibition of the plant extracts was measured by the Griess assay while cytotoxicity were measured by the MTT assay against CEM-SS cell line. The antibacterial bioassay (against 4 bacteria, i.e. Bacillus subtilis B28 (mutant), Bacillus subtilis B29 (wild-type), Pseudomonas aeruginosa UI 60690 and methicillin resistant Staphylococcus aureus, (MRSA) was also carried out using the discdiffusion method.

All tested extracts exhibited very strong antioxidant properties when compared to Vitamin E (Ŭ-tocopherol) with percent inhibition of $89 \ddot{1} 98 \%$ in the FTC and $60 \ddot{1} 95 \%$ in the TBA assays. In the DPPH method, $\mathrm{H}$. herbacea exhibited the strongest radical scavenging activity with an IC50 value of $32 \mathrm{gg} / \mathrm{ml}$. The results from the Griess assay showed that the tested extracts are weak inhibitors of NO synthase. However, all tested extracts exhibited moderate cytotoxic properties against CEM-SS cell line giving CD50 values in the range of $21 \ddot{1} 41 \mathrm{gg} / \mathrm{ml}$. In the antibacterial bioassay, the stems and the roots of $\mathrm{H}$. capitellata showed moderate activity against the 4 tested bacteria while the leaves showed moderate activity towards B. subtilis B28, MRSA and P. aeruginosa only. The roots of H. dichotoma showed strong antibacterial activity against all 4 bacteria. All other extracts did not exhibit any antibacterial activity.
\end{abstract}

Keyword: Antioxidant; Radical scavenging; Anti-inflammatory; NO inhibition; Cytotoxic; Antibacterial; Hedyotis 